\title{
Special Non-Finite Forms of Latin and Albanian
}

\author{
Dr. Leonard Xhamani
}

Docente di Latino, Università di Tirana

Email: leonardxhemani@hotmail.com

\section{Doi:10.5901/jesr.2014.v4n4p373}

\begin{abstract}
The verbal system of Latin language, just like that of Albanian, apart from the finite forms, is characterized by a number of nonfinite forms, which make this system more complete and diverse. In this speech, we will be focused on a special non-finite form of Latin, that of supinum, which has no relevant structure in Albanian. We will see the supinum according to its way of formation and its noun and verb features. The examples that illustrate this non-finite form of Latin will be translated, in order to have a better understanding of the verbal forms of Albanian which correspond to it. An important part in this speech will be occupied by the Albanian non-finite forms, of the type me të ardhur dhe një ardhur, observing not only their way of formation and the function they have, but also how they can be expressed in Latin. However, according to their structural type and grammatical values, these two forms have no relevant ones in Latin, but they can be expressed by some structures including the participle. The method used is that of the approach, a well- known method in Linguistics, which through the comparison of different elements, not only within a language, but also between different languages, permits the analyses and the comparative outlook.
\end{abstract}

Keywords: Latin and Albanian language, non-finite forms, supinum, me të ardhur and një të ardhur.

\section{Introduzione allo Studio}

Il sistema verbale della lingua latina, al pari di quello della lingua albanese, oltre alle forme esplicite, viene caratterizzato anche da un numero di forme implicite, con le quali questo sistema diventa più completo e variegato. La lingua latina ha cinque forme implicite che sono: infinitivus, participium, gerundium, gerundivium e supinum. Nelle opere degli scrittori antichi queste forme si usano con frequenza, specialmente l'infinito ed il participio, che vengono contraddistinti da una molteplicità di forme morfologiche e funzioni sintattiche. Ma anche le altre forme non rimangono indietro per quanto riguarda l'uso e le loro caratteristiche. Dall'altra parte, queste forme implicite sono presenti anche nella lingua albanese. Occorre aggiungere che nella nostra lingua tali forme hanno seguito una via tutta loro per quanto riguarda le modalità di costruzione. Dall'antica fase indoeruopea l'albanese ha ereditato solamente il participio passato con significato passivo (Demiraj, 1976: 22). La lingua albanese nel corso della sua evoluzione storica ha creato anche un numero di forme implicite sulla base del participio sostantivato. Alcune di queste forme implicite hanno assunto valori grammaticali che corrispondono in modo generale alle forme implicite della lingua latina. Qui possiamo menzionare l'infinito gegë del tipo me bam, il quale ha assunto i valori grammaticali che corrispondono a quelli dell'infinito latino, anche se questo infinito dell'albanese è rimasto solo a livello dialettale. Nell'albanese standard questa forma non si usa, poiché il suo posto è stato occupato dal modo congiuntivo e dalla forma del tipo për të bërë, denominata anche il secondo infinito; quest'ultimo ha assunto valori grammaticali che corrispondono in parte all'infinito latino ed in parte al supino ed al gerundio; la forma del tipo duke bërë corriponde in parte all'infinito latino ed in parte al gerundio; la forma del tipo pa bërë dal punto di vista strutturale non ha corrispondenze nella lingua latina, ma una forma simile si esprime con forme diverse, tra le quali possiamo menzionare le costruzioni con sine + ablativo, in casi particolari non + participio, ecc; la forma del tipo me të ardhur e një të ardhur sia dal punto di vista strutturale, sia dal punto di vista dei valori grammaticali, non ha corrispondenze, ma questa forma si può esprimere con altre costruzioni tra cui il participio.

In questo studio ci concentreremo sulle forme implicite particolari delle due lingue: il supino del latino e le forme implicite del tipo me të ardhur e një të ardhur dell'albanese. II supino latino verrà analizzato nella modalità di costruzione, nelle sue caratteristiche verbali e sostantivali e troveremo inoltre anche la forma dell'albanese alla quale corrisponde. Gli esempi illustrativi per questa forma implicita verranno tradotti in albanese, per capire meglio quali sono le forme verbali corrispondenti nella nostra lingua. Per quanto riguarda le forme implicite dell'albanese del tipo me të ardhur e një të ardhur occorre dire che verranno trattate dal punto di vista della costruzione e delle funzioni che hanno, vedremo inoltre come si possono esprimere queste forme nella lingua latina. 


\section{II Supino della Lingua Latina}

Nel gruppo delle forme implicite che combinano caratteristiche verbali e nominali, oltre all'infinito ed il gerundio, si include anche il supino. II supino è un sostantivo verbale d'azione della quarta declinazione, del genere maschile che si usa solo nel caso accusativo e ablativo del numero singolare e ha le terminazioni - um ed $-u$. Fa parte del paradigma del verbo e serve per formare il participio passato passivo, il participio futuro attivo e l'infinito del futuro passivo dei verbi (per la modalità di costruzione del participio e dell'infinito si veda la tesi del dottorato di ricerca: Xhemani L., Format e pashtjelluara të latinishtes dhe të shqipes (Vështrim krahasues). Nella lingua latina, entrambe le forme hanno un uso molto limitato e possono essere sostituite sempre con altre costruzioni. II supino può essere, come è stato detto, di due forme: attivo e passivo. (La vecchia denominazione del supino attivo o passivo, ci sembra giusto, poiché il valore attivo e passivo è una caratteristica non solo dei verbi, ma anche degli aggettivi. Si noti che il supino attivo regge un complemento oggetto anche quando si collega a iri - infinito impersonale ed eo - nella formazione dell'infinito futuro passivo. L'espressione "spero urbem captum iri" grammaticalmente ha il valore "shpresoj që të shkohet të merret qyteti". Dall'altra parte i supini con $-u$ appartengono a tutti i verbi transitivi ed intransitivi che hanno realmente valore passivo, ad eccezione degli arcaismi "cubĭtu surgěre" e "obsonatu redire" dove le due forme, dopo aver perso l'idea verbale, devono essere considerati dei veri ablativi disgiuntivi.)

Il caso accusativo, con la terminazione -um, ha il valore del supino attivo.

Laudat-um për të lavdëruar

Monit-um për të qortuar

Lect-um për të lexuar

Audit-um për të dëgjuar

Il caso accusativo del supino si usa dopo i verbi che indicano movimento ed esprime lo scopo dell'azione. In questo caso il supino può reggere anche un complemento diretto. Nella lingua albanese, il supino in questo caso si può tradurre con un infinito del tipo për të punuar. Per esempio:

$>$...ipse DC cum equitibus magnis itineribus progreditur edictum que praemittit, ad quam diem magistratus principesque omnium civitatum sibi esse praesto Cordubae vellet.

- Vetë ai me 600 kalorës niset për një rrugë të gjatë dhe dërgon përpara dikë për të njoftuar se në cilën ditë magjistratët dhe paria e qyteteve duhej të ishte në pritje të tij në Kordubë. (Caes. Liber II, kap. 19).

> His pontibus pabulatum mittebat, quod ea quae citra flumen fuerant, superioribus diebus consumpserat.

- Nga këto ura dërgonte ushtarë për të marrë ushqim për kafshët, sepse ajo që ishte këtej lumit, e kishte harxhuar gjatë ditëve të para.

$>$ Neque civitates, quae ad Caesaris amicitiam accesserant, frumentum supportare, neque ei, qui pabulatum longius progressi erant, interclusi fluminibus reverti neque....

- As popullsitë që kishin mbajtur anën e Çezarit, nuk sillnin dot grurë, as ata që kishin shkuar edhe më larg për të marrë ushqim, të penguar nga lumenjtë, nuk mund të ktheheshin... (Caes. Liber I, kap. 48).

$>$ Proximo die praesidio in castris relicto universas ad aquam copias educunt, pabulatum emittitur nemo.

- Ditën tjetër (të nesërmen), pasi lënë roje një pjesë në kamp, i dërgojnë të gjithë për të siguruar ujë; për të siguruar ushqim, nuk dërgohet asnjë. (Caes. Liber I, kap. 81).

$>$...et cum eo L. Vargunteius senator constituere ea nocte paulo post cum armatis hominibus sicuti salutatum introire ad Ciceronem ac de inproviso domi suae inparatum confodere.

- ...dhe bashkë me senator Varguntein vendosën që po atë natë, pak më vonë, me disa njerëz të armatosur, të hynin te Cicëronit sikur për ta përshëndetur dhe ta vrisnin atë papritur në shtëpinë e tij. (Sal, kap. XXVIII).

$>$...ne illi sanguinem nostrum largiantur et, dum paucis sceleratis parcunt, bonos omnis perditum eant!

- ...të mos tregohen zemërgjerë me gjakun tonë dhe, ndërsa kursejnë ca kriminelë, të mos shkatërrojnë tërë qytetarët e ndershëm! (Sal. kap. LII).

$>$ Haedui legatos ad Caesarem mittunt rogatum auxilium.

- Eduinët dërguan ambasadorë te Çezari, për të kërkuar ndihmë. (Caes.)

$>$ Bocchus legatos Romam miserat amicitiam petitum.

- Boko kishte dërguar ambasadorë në Romë për të kërkuar miqësi. (Sal)

$>$ Cubitum discessimmus. - Shkuam për të fjetur.

Il supino attivo si usa anche nelle espressioni:

> nuptum dare filian (nuptum collocare) - jap vajzën për grua (Hostis est uxor quae invita ad virum nuptum datur)

$>$ sessum recipere - bëj vend për të ulur. 
Occorre dire che non tutte le proposizioni finali che dipendono dai verbi di movimento si possono esprimere con il supino. I supini attivi usati nella prosa classica sono esigui (Tantucci, 1992: 372). Tra i più frequenti possiamo menzionare: ambulatum (për të shëtitur, marshuar), cubitum (për të fjetur), deductum (për të drejtuar), defensum (për të mbrojtur), deliberatum (për të gjykuar, vendosur), deprecatum (për të lutur, shmangur), dormîtum (për të fjetur), edictum (për të njoftuar), emptum (për të blerë), exsulatum (për të gëzuar), frumentatum (për të mbledhur drithë), habitatum (për të banuar), hiematum (për të dimëruar), laudatum (për të lavdëruar), nuptum (për të martuar), obsecratum (për të lutur), oppugnatum (për të sulmuar), oratum (për të folur), pabulatum (për të marrë ushqim), perditum (për të humbur), petitum (për të kërkuar), ploratum (për të vajtuar), postulatum (për të kërkuar), rogatum (për të pyetur), salutatum (për të përshëndetur), sessum (për të ulur), ecc.

Il caso ablativo, con la terminazione $-u$, ha il valore del supino passivo.

Laudat-u për t'u lavdëruar

Monit-u për t'u qortuar

Lect-u për t'u lexuar

Audit-u për t'u dëgjuar

Hanno supino passivo solo i verbi transitivi, inclusi i deponenti, che in questa forma hanno un significato passivo. II supino passivo si usa in dipendenza dagli aggettivi (usati di più come neutri), quali: acerbus (i ashpër, i parakohshëm), facilis (i lehtë), difficilis (i vështirë), incredibilis (i pabesueshëm), iucundus (i këndshëm), horribilis (i tmerrshëm), pulcher (i bukur), utilis (i dobishëm), ecc., con il valore dell'ablativo di limitazione. Nella lingua albanese il supino di questo caso si può tradurre con l'infinito passivo del tipo për t'u punuar. Per esempio:

> Ubi de magna virtute atque gloria bonorum memores, quae sibi quisque facilia factu putat, aequo animo accipit,...

- Kur kujton virtytin e madh dhe lavdinë e njerëzve të ndershëm, presin me indiferencë ato që çdonjeri i mendon të lehta për t'u bërë edhe prej vetë atij,... (Sal. kap. III).

$>$ In tanta tamque corrupta civitate Catilina, id quod factu facillumum erat, omnium flagitiorum atque facinorum circum se tamquam stipatorum catervas habebat.

- Në një qytet aq të madh dhe aq të korruptuar, Katilina, gjë që ishte mjaft e lehtë për t'u bërë, mblodhi rreth vetes një tufë njerëzish të të gjitha krimeve dhe poshtërsive. (Sal. kap. XIV).

- Erat vallis inter duas acies, ut supra demonstratum est, non ita magna, at difficili et arduo ascensu.

- Ndërmjet dy ushtrive ndodhej një luginë, siç e kemi treguar më lart, jo shumë e madhe, por e vështirë dhe e rrëpirët për t'u ngjitur. (Caes. Liber II, kap. 34).

$>$ Hi postquam in una moenia convenere, dispari genere, dissimili lingua, alii alio more viventes, incredibile memoratu est, quam facile coaluerint.

- Këta pasi u mblodhën brenda të njëjtave mure, ndonëse ishin të racave të ndryshme, me gjuhë të ndryshme dhe secili jetonte me zakonet e veta, u bashkuan aq lehtë, sa është e pabesueshme për t'u thënë. (Sal. kap. $\mathrm{VI})$.

$>$ Sed civitas incredibile memoratu est adepta libertate quantum brevi creverit.

- Por qyteti, është e pabesueshme për t'u thënë, arriti një përparim brenda një kohe të shkurtër. (Sal. kap. VII).

I supini passivi più usati nella prosa classica sono: dictu (për t'u thënë), factu (për t'u bërë), audïtu (për t'u dëgjuar), visu (për t'u parë), cognĭtu (për t'u njohur), memorātu (për t'u kujtuar), adspectu (për t'u shikuar, parë), intellectu (për t'u kuptuar), inventu (për t'u sajuar), gustatu (për t'u shijuar), perpessu (për t'u duruar), probatu (për t'u shqyrtuar), tactu (për t'u prekur), toleratu (për t'u duruar), scitu (për t'u ditur). Per esempio:

$>$ Rem non modo visu foedam, sed etiam auditu! (Cic.)

- Gjë e turpshme jo vetëm për t'u parë, por edhe për t'u dëgjuar.

$>$ Nihil dignum memoratu actum est. - Nuk u bë asgjë e denjë për t'u kujtuar.

Come abbiamo già detto sopra, il supino è quella forma implicita che si usa raramente. Al posto del supino con $-u$ si possono usare altre costruzioni. I verbi che non hanno la forma del supino, possono usare al suo posto l'infinito oppure il gerundio. Per esempio:

- Rruga nuk është e lehtë për t'u gjetur.

Al posto di: Via haud facilis est inventu.

Si può dire: Haud est facile invenire viam.

Mentre per la frase:

- Këto gjëra janë shumë të lehta për t'u gjykuar",

Non essendo in uso il supino iudicatu, bisogna usare:

Haud est difficile iudicare haec, ose Haec ad iudicandum sunt facillima. 


\section{La Forma Implicita del Tipo Me të Ardhur e Del Tipo Një të Ardhur}

Nella lingua albanese, oltre a quelle già conosciute, si incontrano anche altre forme implicite, quelle del tipo me të ardhur e një të ardhur. II primo sintagma viene costruito dalla particella prepositiva me e dal sostantivo verbale neutro del tipo të ardhur, dove le parti costituenti hanno perso sempre di più la loro autonomia (Per la modalità di costruzione di questo sintagma vedasi Demiraj, 1986: 977-986). Oltre alla categoria grammaticale della diatesi, questa forma implicita ha anche un significato temporale condizionato. Denomina un'azione che si compie o che si aspetta si possa compiere subito prima dell'azione espressa dal verbo principale. Dunque, questa forma si accompagna da una forma verbale esplicita, la quale di solito si mette in uno dei tempi del passato, e dipende da essa. Le costruzioni con questo sintagma grammaticalizzato si possono intercambiare con altre costruzioni esplicite. Quando il verbo principale è in uno dei tempi del passato o nel presente dell'indicativo, allora questo sintagma si può intercambiare con lo stesso tempo del verbo rispettivo. Questa costruzione, come viene messo in evidenza dai linguisti, è sinonimica alla proposizione temporale della posteriorità (Çeliku, 1989: 44). In ogni caso la forma esplicita che ha sostituito il sintagma del tipo me të ardhur, viene preceduto dalle congiunzioni: sa, sapo, posa (Akademia e Shkencave: 342). Per esempio:

- Asaj, me të marrë vesh (sapo kishte marrë vesh) punën e re, i kishte hipur një ndezulli e pazakontë. (Kadare, Spiritus, f. 205).

- Me të dëgjuar (sapo dëgjoi) ringjalljen e historisë së kapjes së një fantazme prej Sigurimit Shqiptar, histori në të cilën kapësi merret me mend, zinte një vend qendror, e sidomos, kur u pohua njëzëri se kapësi pas nderimeve të para, rritje në gradë, dekorim me Urdhrin e Flamurit etj., një vit më pas u godit nga mallkimi dhe u dënua dyfish prej shtetit dhe prej qiellit, domethënë me burg dhe me gjymtim trupor, me t'u përhapur (sapo u përhap), pra, pëshpërima e vjetër, aty për aty, disa ish-të burgosur u kujtuan se e kishin takuar të gjymtuarin në burg ose në kamp. (Kadare, Spiritus, f. 244).

- Në një rast tjetër hetuesi, me të dëgjuar (sapo të dëgjonte) se dëshmitari e merrte në telefon, do të ngrihej, natë e mesnatë qoftë, i vetëm a me grua në shtrat. (Kadare, E penguara, f. 50).

La gramaticalizzazione di questo sintagma è, senza dubbio, più recente rispetto a quella del tipo për të bërë, poiché non si scontra nei nostri vecchi autori. Secondo i nostri linguisti, la grammaticalizzazione del sintagma in questione sarà stata eseguita verso il XVI-esimo secolo.

Una tale forma implicita non si conosce nella lingua latina. Ma questa forma implicita si può esprimere con il participio, oppure per i verbi che ne sono carenti, con una proposizione subordinata con forma verbale esplicita. In questi casi, il participio è parte dell'ablativo assoluto. Per esempio:

$>$ At Pompeius cognitis his rebus, quae erant Orici atque Apolloniae gestae, Dyrrachio timens diurnis eo nocturnisque itineribus contendit...

- Pompeu nga ana e tij, me të mësuar se ç'kishte ndodhur në Orikum dhe në Apoloni, duke u frikësuar për Dyrrahun, u drejtua për atje, duke marshuar ditë e natë. (Caes. Liber III, kap. 13).

> Cuius adventu cognito diffisus municipii voluntati Thermus cohortes ex urbe reducit et profugit.

- Me të marrë vesh mbërritjen e tij, Termi, duke mos pasur besim te qëndrimi i banorëve, nxjerr kohortat nga qyteti dhe largohet me nxitim. (Caes. Liber I, kap. 12).

$>$ Hoc tumultu nuntiato Marcellinus cohortes subsidio nostris laborantibus submittit ex castris.

- Me t'u njoftuar për këtë mësymje, Marcelini nisi disa kohorta nga kampi, për të ndihmuar tanët që ishin në rrezik. (Caes. Liber III, kap. 64).

Nei casi in cui il participio di un verbo intransitivo attivo come «venire - vij, exire - dal, redire - kthej», non hanno il participio passato, quei verbi si possono dare con cum + congiuntivo, oppure, se hanno valore qualificativo, con una proposizione congiuntiva. Per esempio:

$>$ Cum Caesar Romam venisset... - Çezari, me të ardhur në Romë...

$>$ Legati, qui Romam veněrant, magnis honoribus accepti sunt.

- Ambasadorët, me të ardhur në Romë, u pritën me nderime të mëdha.

Per quanto riguarda il sintagma një të ardhur, come quello sopra, è stato costruito dalla particella një, che non si usa più come numerale, e dal sostantivo verbale neutro del tipo të ardhur (Demiraj, 1986: 988). Dunque, entrambe le parti costituenti hanno perso la loro autonomia; il primo elemento non ha più il valore del numerale per indicare quantità $\mathrm{e}$ nemmeno il secondo elemento ha il valore del sostantivo. Essi esprimono un unico significato, denominando un'azione come processo, esattamente come le altre forme implicite. La sua cristallizzazione come forma implicita, come dice il prof. Kolec Topalli, «dimostrano la possibilità di reggere complemento diretto (një të filluar punën) oppure una particella pronominale (një ta parë) e la possibilità di trasformare il verbo dalla diatesi attiva in quella passiva tramire la particella $u$ (një t'u larë)» (Topalli, 2011: 1084). Questo sintagma si può usare con lo stesso valore di quello del tipo me të ardhur. In 
questo caso si può intercambiare con esso, specialmente se vengono usati con la funzione temporale. Possiamo dire che il sintagma in questione si usa di più nella lingua parlata e molto raramente in quella scritta. Per esempio: (Akademia e Shkencave, 342):

- Të lumtë, o Leks, një të ikur unë nga shtëpia, një t'u shthurur gardhi.

- Një të parë atë, sakaq, edhe nxënësit e tjerë filluan të lëviznin.

Una tale forma implicita si può esprimere nella lingua latina con il participio, con gli stessi esempi che abbiamo precedentemente dato per il sintagma del tipo me të ardhur. Comunque possiamo dire che questa forma non l'abbiamo scontrata nelle traduzioni delle opere prese in esame, poiché al suo posto è stato usato il primo sintagma me të ardhur.

\section{Conclusioni}

Il supino è quella forma implicita, in cui si intrecciano caratteristiche verbali e sostantivali. Esso serve per formare alcune altre forme implicite e alcune forme composte del verbo. II supino è un sostantivo verbale della quarta declinazione che si usa nel caso accusativo e ablativo singolare. II caso accusativo ha la terminazione -um ed un significato attivo, mentre l'ablativo ha la terminazione $-u$ ed ha un significato passivo. II caso accusativo del supino si usa dopo i verbi che indicano movimento ed esprime lo scopo dell'azione. In questo caso il supino può reggere anche un complemento diretto. Nella lingua albanese, il supino di questo caso si può tradurre con l'infinito del tipo për të punuar. II caso ablativo del supino si incontra solo nei verbi transitivi e quelli deponenti e si usa in dipendenza da alcuni aggettivi. Nella lingua albanese il supino di questo caso si traduce con l'infinito passivo del tipo për t'u punuar. Nella lingua latina, entrambe le forme del supino, hanno un uso limitato e si possono sostituire da altre costruzioni.

L'albanese durante la sua evoluzione interna, accanto alle forme implicite come, il participio, l'infinito, il gerundio e la forma negativa, ha creato anche altre forme implicite del tipo me të ardhur e një të ardhur. Queste forme, oltre alla categoria grammaticale della diatesi, hanno anche un significato temporale condizionato. Esse si possono intercambiare con una forma esplicita del verbo, preceduta dalle congiunzioni sa, sapo, posa. Forme implicite simili non si scontrano nella lingua latina. Comunque possiamo dire che queste costruzioni si possono esprimere o tramite il participio oppure con la forma esplicita.

\section{References}

Akademia e Shkencave, Instituti i Gjuhësisë dhe i Letërsisë (1995), Gramatika e gjuhës shqipe, I, Tiranë.

Çeliku, M. (1989). Ndërtimet e tipit me të ardhur dhe një të ardhur, Tiranë: Gjuha Jonë, 2.

Çeliku, M. (2006). Format e pashtjelluara të foljes në gjuhën e sotme shqipe, Tiranë: Shblu.

De Giorgi, M. \& Mandressi, P. (1999). I verbi latini, Milano: Hoepli.

Demiraj, Sh. (1976). Morfologji historike e gjuhës shqipe, (parte II), Tiranë: Mihal Duri.

Demiraj, Sh. (1986). Gramatikë historike e gjuhës shqipe, Tiranë: 8 Nëntori.

Diotti, A. (2000). Lexis 2, Roma: Bruno Mondadori.

Famerie, E. \& Bodson, A. \& Dubuisson, M. (2012). Methode de langue latine, Paris: Armand Colin.

McKeown, Jc. (2010). Classical Latin, Indianapolis: Hackett Publishing.

Tantucci, V. (1992). Urbis et orbis lingua, Bologna: Poseidonia.

Terracina, F. (2003). Verbi latini, Tutti i verbi regolari e irregolari, Milano: Vallardi.

Topalli, K. (2011). Gramatikë historike e gjuhës shqipe, Tiranë: Botimet Albanologjike.

Xhemani, L. (2012). Format e pashtjelluara të latinishtes dhe të shqipes (Vështrim krahasues), tesi di dottorato di ricerca. 